\title{
Tendência temporal da mortalidade neonatal no Estado do Pará entre 2010 e 2019
}

\author{
Temporal trend of neonatal mortality in the State of Pará between 2010 and 2019 \\ Tendencia temporal de la mortalidad neonatal en el Estado de Pará entre 2010 y 2019
}

Recebido: 18/10/2021 | Revisado: 10/10/2021 | Aceito: 20/10/2021 | Publicado: 22/10/2021

\author{
Rosimeire da Silva Morais Rezende \\ ORCID: https://orcid.org/0000-0001-7590-8441 \\ Faculdade de Ciências Sociais Interamericana, Brasil \\ E-mail: rosemeiresmr@hotmail.com \\ Dilma Germana de Abreu Pereira \\ ORCID: https://orcid.org/0000-0002-4713-4183 \\ Faculdade de Ciências Sociais Interamericana, Brasil \\ E-mail: dilmagermana@gmail.com \\ Leonardo Morais Rezende dos Santos \\ ORCID: https://orcid.org/0000-0001-6108-3055 \\ Universidad María Serrana, Paraguai \\ E-mail: leonardomoarisd50@gmail.com \\ Joanny Silva Moraes \\ ORCID: https://orcid.org/0000-0002-5605-1566 \\ Universidade Federal do Norte do Tocantins, Brasil \\ E-mail: Joannymoraes@gmail.com \\ Clessia Alessandra Rezende dos Santos \\ ORCID: https://orcid.org/0000-0003-3696-2001 \\ Hospital Regional de Conceição do Araguaia, Brasil \\ E-mail: clessiaale@hotmail.com \\ Elismara da Silva Seabra \\ ORCID: https://orcid.org/0000-0003-0736-8900 \\ Hospital Regional de Conceição do Araguaia, Brasil \\ E-mail: elismaralila@ hotmail.com
}

\begin{abstract}
Resumo
Ainda é discrepante a mortalidade neonatal entre os Estados Brasileiros, o que é passivo da desigualdade social inerente e estruturado na sociedade, as quais são históricas e muito expressivas, o que reflete na saúde da população. Diante deste exposto, o presente estudo buscou analisar a tendência temporal da mortalidade neonatal no Estado do Pará, durante o período de 2010 a 2019. Trata-se de um estudo ecológico, com caráter quantitativo e de série temporal. Os dados foram obtidos no Sistema de Informações de Mortalidade e no Sistema de Informações sobre Nascidos Vivos. Para avaliar a tendência temporal das séries de mortalidade, foi definida como variável independente o ano amostrado (2010 a 2019), e como variáveis dependentes a região de saúde, sexo e faixa etária da criança, peso da criança ao nascer, cor da pele/raça e idade da mãe, duração da gestação, tipo de gravidez e tipo de parto. A pesquisa identificou um total de 22.560 óbitos neonatais (ON) na população no Estado do Pará no período de 2010 a 2019. A taxa média de $\mathrm{ON}$ foi de 22,6 por 1.000 nascidos vivos (NV), com uma variação média anual ( $\beta$ ) indicando um decréscimo de $-47,7575\left(\mathrm{r}=0,9504 ; \mathrm{R}^{2}=0,9034 ; \mathrm{p}\right.$-valor $=\langle 0,001)$. Os riscos de morte mais elevados de neonatais no Estado do Pará, correspondem à fatores do peso ao nascer de até $500 \mathrm{~g}$, faixa etária da mãe acima de 30 anos e duração da gestação entre 22 e 37 semanas.
\end{abstract}

Palavras-chave: Mortalidade neonatal; Recém-nascido; Fatores de risco; Epidemiologia; Estudo ecológico.

\begin{abstract}
There is still a discrepancy between neonatal mortality among Brazilian States, which is a liability of the inherent and structured social inequality in society, which are historical and very expressive, which reflects on the health of the population. Given this, this study sought to analyze the temporal trend of neonatal mortality in the State of Pará, from 2010 to 2019. This is an ecological study, with a quantitative character and a time series. Data were obtained from the Mortality Information System and the Live Birth Information System. To assess the temporal trend of the mortality series, the year sampled (2010 to 2019) was defined as an independent variable, and as dependent variables the region of health, sex and age of the child, weight of the child at birth, skin color/ race and age of the mother, duration of pregnancy, type of pregnancy and type of delivery. The survey identified a total of 22,560 neonatal deaths (ND) in the population in the State of Pará from 2010 to 2019. The average rate of ND was 22.6 per 1000 live births (LB), with an average annual variation $(\beta)$ indicating a decrease of $-47.7575\left(\mathrm{r}=0.9504 ; \mathrm{R}^{2}=0.9034 ; \mathrm{p}\right.$-value $\left.=<0.001\right)$. The highest risks of death for newborns in the State of Pará correspond to factors such as birth weight up to $500 \mathrm{~g}$, mother's age group above 30 years and gestation duration between 22 and 37 weeks.
\end{abstract}

Keywords: Neonatal mortality; Newborn; Risk factors; Epidemiology; Ecological study. 


\section{Resumen}

Aún existe una discrepancia entre la mortalidad neonatal entre los estados brasileños, que es un pasivo de la desigualdad social inherente y estructurada en la sociedad, que son históricas y muy expresivas, que se reflejan en la salud de la población. Ante esto, este estudio buscó analizar la tendencia temporal de la mortalidad neonatal en el Estado de Pará, de 2010 a 2019. Se trata de un estudio ecológico, de carácter cuantitativo y una serie de tiempo. Los datos se obtuvieron del Sistema de Información de Mortalidad y del Sistema de Información de Nacidos Vivos. Para evaluar la tendencia temporal de la serie de mortalidad, se definió como variable independiente el año muestreado (2010 a 2019), y como variables dependientes la región de salud, sexo y edad del niño, peso del niño al nacer, color de piel / raza y edad de la madre, duración del embarazo, tipo de embarazo y tipo de parto. La encuesta identificó un total de 22.560 muertes neonatales (ON) en la población del Estado de Pará de 2010 a 2019. La tasa promedio de ON fue de 22,6 por cada 1.000 nacidos vivos (BR), con una variación anual promedio ( $\beta$ ) que indica una disminución de 47,7575 ( $\mathrm{r}=0,9504 ; \mathrm{R}^{2}=0,9034$; valor de $\left.\mathrm{p}=<0,001\right)$. Los mayores riesgos de muerte para los recién nacidos en el estado de Pará corresponden a factores como peso al nacer de hasta $500 \mathrm{~g}$, grupo de edad de la madre mayor de 30 años y duración de la gestación entre 22 y 37 semanas.

Palabras clave: Mortalidad neonatal; Recién nacido; Factores de riesgo; Epidemiología; Estudio ecológico.

\section{Introdução}

A mortalidade neonatal é considerada um dos melhores indicadores do nível de vida e bem-estar social de uma população, devido a expressiva e complexa conjunção de fatores biológicos, socioeconômicos e assistenciais envolvidos com este fenômeno (Soares \& Menezes, 2010). Compreende-se por mortalidade neonatal os óbitos ocorridos entre 0 e 27 dias de vida, o qual se subdivide em precoce (ocorre na primeira semana de vida) e tardia (ocorre entre o sétimo e $27^{\circ}$ dia de vida) (Gaiva et al., 2015). A Organização Mundial de Saúde (OMS) afirma que a taxa de mortalidade infantil permite analisar a disponibilidade, a utilização e a eficácia dos cuidados de saúde, em especial da atenção ao pré-natal, ao parto, ao recémnascido e a criança no primeiro ano de vida, sendo frequentemente utilizada para definir políticas direcionadas à saúde materno-infantil (Brasil, 2002; França \& Lansky, 2016).

Atualmente, as menores taxas de mortalidade neonatal ocorrem em países com elevados Índices de Desenvolvimento Humano (IDH), enquanto os países com baixo IDH apresentam as maiores taxas de mortalidade, evidenciando uma estreita relação destas mortalidades com elementos de desenvolvimento humano, assim como do sistema de saúde, que por muitas vezes são ineficientes em países menos desenvolvidos (Kropiwiec et al., 2017). Estimativas globais apontam uma morte neonatal para cada 1000 nascimentos no Japão e Irlanda, enquanto em países com IDH mais baixos como Mali, Serra Leoa e Somália esta proporção é de 48 mortes a cada 1000 nascidos vivos (Walker et al., 2013). O Ministério da Saúde (MS) criou em 2011, uma rede de atenção para garantir acesso e resolutividade durante o pré-natal, parto e período neonatal (Rede Cegonha). Com esse modelo de atenção, o governo pretendeu fortalecer a rede hospitalar obstétrica de alto risco e ampliar o número de leitos do Sistema Único de Saúde (SUS), além de qualificar os profissionais de saúde para o atendimento à gestante e ao neonato (Brasil, 2011).

Em 2011, o Brasil alcançou um marco na história do país, contemplando um dos objetivos traçados no projeto de Objetivos de Desenvolvimento do Milênio, o qual faz parte de um compromisso dos governos integrantes das Organizações das Nações Unidas para a redução da mortalidade infantil (Veloso et al., 2019). Apesar dessa redução nas mortalidades neonatais no Brasil ao longo do tempo, ainda há uma discrepância em relação às mortalidades neonatais entre os Estados Brasileiros, principalmente entre os Estados do Norte/Nordeste e os do Sul/Sudeste (Migoto et al., 2018). Está discrepância na mortalidade neonatal entre os Estados Brasileiros, é passivo da desigualdade social inerente e estruturado na sociedade, as quais são históricas e muito expressivas, o que reflete na saúde da população, fazendo com que os determinantes sociais de saúde sejam grandes protagonistas neste contexto (Paim et al., 2011). As variáveis referentes ao ambiente externo e às condições gerais de subgrupos da população, compreendendo assim o nível econômico, a escolaridade, o local de residência, a sexualidade, entre outras variáveis, são fatores determinantes para a saúde pública e indicam a complexidade da interação entre as condições sociais e a utilização dos serviços de saúde, prevalências que afetam os resultados para mortalidade de recém- 
nascidos (Silveira et al., 2008; Viellas et al., 2014).

O Estado do Pará é o segundo maior estado do Brasil em extensão territorial 1.248 .000 km², e sua imensa distribuição geográfica atrelada ao difícil acesso às regiões mais isoladas do estado, torna o acesso à saúde um desafio para o governo local (Pontes et al., 2020). O Instituto Brasileiro de Geografia e Estatística (IBGE, 2021) aponta uma taxa de 15,40 por mil nascidos vivos para o ano de 2017, tornando o Estado o $6^{\circ}$ no Ranking nacional para a taxa de mortalidade infantil (idade menor que 1 ano). Não foi encontrado na literatura estudos apontando o perfil da mortalidade neonatal no Estado do Pará e/ou sua distribuição nas regiões de saúde do Estado, tão pouco as especificidades atreladas aos fatores de riscos para as mortalidades neonatais no Estado Paraense.

Diante deste exposto e com o intuito de contribuir com informações que possibilitem a diminuição das taxas de mortalidade neonatal no Estado do Pará, o presente trabalho parte da problemática de que variáveis relacionadas ao desenvolvimento social e saúde pública, como a interdependência da região de saúde em que está inserida a mãe, idade materna, cor da pele/raça, peso ao nascer e tempo de gestão, podem ser fatores que apresentam forte correlação com a mortalidade neonatal no Estado do Pará? Para responder a questão problema do estudo, objetivou-se analisar a tendência temporal da mortalidade neonatal no Estado do Pará, durante o período de 2010 a 2019, mediante as características de nascidos vivos e óbitos neonatais.

\section{Metodologia}

Trata-se de um estudo ecológico, com caráter quantitativo e de série temporal. A população do estudo correspondeu aos nascidos vivos e óbitos neonatais ocorridos no período de 2010 a 2019 no Estado do Pará. Ademais, informações referentes as mães residentes no estado em questão, também fizeram parte da população de estudo. Os dados foram obtidos no Sistema de Informações de Mortalidade (SIM) e no Sistema de Informações sobre Nascidos Vivos (SINASC), ambos de domínio público disponibilizados pelo Departamento de Informática do Sistema Único de Saúde (DATASUS), com informações sociodemográficas de nascimento e mortalidade da população residente no Estado do Pará.

O material foi selecionado a partir da busca de variáveis potencialmente representativas de aspectos da desigualdade social no Pará, tendo como fonte os registros contidos nas bases de dados mencionadas anteriormente. Para avaliar a tendência temporal das séries de mortalidade, foi definida como variável independente o ano amostrado (2010 a 2019), e como variáveis dependentes a região de saúde, sexo e faixa etária da criança, peso da criança ao nascer, cor da pele/raça e idade da mãe, duração da gestação, tipo de gravidez e tipo de parto.

Após a extração e tabulação das variáveis de interesse, os dados foram transferidos para planilha do Excel, os quais foram formatados em tabelas com a distribuição dos óbitos da população estudada. Em seguida, foi calculado o coeficiente de determinação das séries (R2), contendo a variação anual das médias dos valores das séries ( $\beta$ ), a partir da regressão linear de Pearson, e o valor de p (valor-p) a partir da análise de variância (ANOVA).

Para examinar o comportamento (aumento, queda, estabilidade) da variação média anual do coeficiente de mortalidade neonatal, foi avaliado o valor (positivo ou negativo) e a significância estatística do coeficiente de regressão ( $\beta$ ) (Tomio \& Magajewski, 2019). O coeficiente de mortalidade foi considerado crescente quando $\beta$ mostrou-se positivo, e decrescente quando $\beta$ foi negativo. A significância estatística do modelo foi estabelecida para valor de $\mathrm{p}<0,05$.

O estudo realizado foi do tipo ecológico e, portanto, sem sujeitos de pesquisa, mas agregados populacionais de análise. Os bancos de dados utilizados são de domínio e acesso públicos e sem informações sobre a identidade dos participantes ou qualquer informação pessoal que permita a identificação ou coloque em risco o sigilo dos dados. Nos termos da Resolução 510/2016 Artigo 1º Parágrafo Único Incisos II, III e V, esta pesquisa não se enquadrou nas normas do Conselho Nacional de Ética em Pesquisa - CONEP para registro e avaliação pelo sistema CEP/CONEP. Portanto, o autor declara ciência 
do teor da Resolução 466/2012 do Conselho Nacional de Saúde, que serviu de referência para as decisões no âmbito ético para execução do projeto.

\section{Resultados}

A pesquisa identificou um total de 22.560 óbitos neonatais $(\mathrm{ON})$ na população no Estado do Pará no período de 2010 a 2019. A taxa média de ON foi de 22,6 por 1.000 nascidos vivos (NV), com uma variação média anual ( $\beta$ ) indicando um decréscimo de $-47,7575\left(\mathrm{r}=0,9504 ; \mathrm{R}^{2}=0,9034\right.$; $\mathrm{p}$-valor $\left.=<0,001\right)$ (Tabela 1). Em relação as regiões de saúde do Estado do Pará, houve tendência de redução na região do Carajás $(\beta=-4,3818)$, Lago de Tucuruí $(\beta=-6,5515)$, Metropolitana $\mathrm{I}(\beta=$ 20,2182), Metropolitana II $(\beta=-0,6666)$, Metropolitana III $(\beta=-7,3515)$, Rio Caetés $(\beta=-0,3567)$, Tapajós $(\beta=-0,0003)$, Tocantins $(\beta=0,5340)$, Xingu $(\beta=-0,4653)$ e Marajó I $(\beta=-0,4423)$ (Tabela 1). Quanto as regiões que apresentaram tendência de aumento na mortalidade neonatal, destaca-se as regiões de saúde do Araguaia $(\beta=-0,2031)$, Baixo Amazonas $(\beta$ $=-0,1874)$ e Marajó II $(\beta=-0,0599)$ (Tabela 1$)$.

Tabela 1. Tendência temporal da mortalidade neonatal no Estado do Pará entre 2010 e 2019, em função das regiões de saúde.

\begin{tabular}{|c|c|c|c|c|c|c|}
\hline Regiões de saúde & TM & $\mathrm{r}$ & $\mathrm{R}^{2}$ & $\beta$ & p-valor & Tendência \\
\hline Araguaia & 1,29 & 0,4507 & 0,2031 & 1,7879 & 0,191 & Aumento \\
\hline Baixo Amazonas & 2,74 & 0,4329 & 0,1874 & 2,7576 & 0,211 & Aumento \\
\hline Carajás & 2,39 & $-0,6637$ & 0,4404 & $-4,3818$ & 0,036 & Redução \\
\hline Lago de Tucuruí & 1,12 & $-0,8056$ & 0,6490 & $-6,5515$ & 0,005 & Redução \\
\hline Metropolitana I & 4,98 & $-0,9389$ & 0,8815 & $-20,2182$ & $<0,001$ & Redução \\
\hline Metropolitana II & 0,92 & $-0,8164$ & 0,6666 & $-3,0121$ & 0,004 & Redução \\
\hline Metropolitana III & 2,05 & $-0,8162$ & 0,6662 & $-7,3515$ & 0,004 & Redução \\
\hline Rio Caetés & 1,35 & $-0,5972$ & 0,3567 & $-3,3152$ & 0,068 & Redução \\
\hline Tapajós & 0,76 & $-0,0173$ & 0,0003 & $-0,0788$ & 0,962 & Redução \\
\hline Tocantins & 2,00 & $-0,7307$ & 0,5340 & $-4,3515$ & 0,016 & Redução \\
\hline Xingu & 1,14 & $-0,6821$ & 0,4653 & $-2,9455$ & 0,030 & Redução \\
\hline Marajó I & 0,68 & $-0,6651$ & 0,4423 & $-0,7697$ & 0,036 & Redução \\
\hline Marajó II & 1,15 & 0,2448 & 0,0599 & 0,6727 & 0,495 & Aumento \\
\hline Pará & 22,6 & 0,9504 & 0,9034 & $-47,7575$ & $<0,001$ & Redução \\
\hline
\end{tabular}

$\mathrm{TM}$ = taxa média de óbitos neonatais por 1.000 nascidos vivos; $\mathrm{r}=$ coeficiente de correlação de Pearson; $\mathrm{R}^{2}=$ coeficiente de regressão linear; $\beta$ intercepto da reta X com o eixo Y da regressão linear; p-valor = nível de significância da regressão linear. Fonte: Sistema de Informações sobre Mortalidade - SIM.

Ao distribuir a mortalidade neonatal por sexo e faixa etária da criança, observa-se que a tendência da mortalidade foi no geral reduzida, com a maior redução em crianças do sexo masculino $(\beta=-31,1697)$ e na faixa etária de 0 a 6 dias $(\beta=-$ 27,8303). As menores reduções na mortalidade neonatal foram observadas para o sexo feminino $(\beta=-16,6000)$, e para a faixa etária de 7 a 27 dias $(\beta=-7,3576)$ e 28 a 364 dias $(\beta=-12,5758)$ (Tabela 2$)$. 
Tabela 2. Tendência temporal da mortalidade neonatal no Estado do Pará entre 2010 e 2019, em função do sexo e da faixa etária da criança.

\begin{tabular}{lccccc}
\hline Sexo & $\mathrm{r}$ & $\mathrm{R}^{2}$ & $\beta$ & $\mathrm{p}$-valor & Tendência \\
\hline Masculino & $-0,9389$ & 0,8815 & $-31,1697$ & $<0,001$ & Redução \\
Feminino & $-0,8736$ & 0,7631 & $-16,6000$ & $<0,001$ & Redução \\
\hline Faixa etária & $\mathrm{r}$ & $\mathrm{R}^{2}$ & $\beta$ & $\mathrm{p}$-valor & Tendência \\
\hline 0 a 6 dias & $-0,8995$ & 0,8091 & $-27,8303$ & $<0,001$ & Redução \\
7 a 27 dias & $-0,7041$ & 0,4957 & $-7,3576$ & 0,0230 & Redução \\
28 a 364 dias & $-0,7853$ & 0,6168 & $-12,5758$ & 0,0071 & Redução \\
\hline
\end{tabular}

$\mathrm{r}=$ coeficiente de correlação de Pearson; $\mathrm{R}^{2}=$ coeficiente de regressão linear; $\beta$ = intercepto da reta $\mathrm{X}$ com o eixo $\mathrm{Y}$ da regressão linear; $\mathrm{p}$ valor = nível de significância da regressão linear. Fonte: Sistema de Informações sobre Mortalidade - SIM.

No que tange a variável peso ao nascer, houve tendência de redução na mortalidade neonatal para crianças que nasceram com peso de 500 a $999 \mathrm{~g}(\beta=-0,1273), 1000$ a $1499 \mathrm{~g}(\beta=-4,5515), 1500$ a $2499 \mathrm{~g}(\beta=-12,8606), 2500$ a $2999 \mathrm{~g}(\beta$ $=-7,4364), 3000$ a $3999 \mathrm{~g}(\beta=-14,7758)$ e $4000 \mathrm{~g}$ ou mais $(\beta=-1,6788)$. O único aumento para a mortalidade neonatal foi observado para recém-nascidos com o peso ao nascer de até $500 \mathrm{~g}(\beta=1,3818)$ (Tabela 3).

Tabela 3. Tendência temporal da mortalidade neonatal no Estado do Pará entre 2010 e 2019, em função do peso da criança ao nascer.

\begin{tabular}{|c|c|c|c|c|c|}
\hline Peso ao nascer & $\mathrm{r}$ & $\mathrm{R}^{2}$ & $\beta$ & p-valor & Tendência \\
\hline Até $500 \mathrm{~g}$ & 0,7610 & 0,5791 & 1,3818 & 0,011 & Aumento \\
\hline 500 a $999 \mathrm{~g}$ & $-0,0190$ & 0,0004 & $-0,1273$ & 0,958 & Redução \\
\hline 1000 a $1499 \mathrm{~g}$ & $-0,7420$ & 0,5505 & $-4,5515$ & 0,014 & Redução \\
\hline 1500 a $2499 \mathrm{~g}$ & $-0,9421$ & 0,8876 & $-12,8606$ & $<0,001$ & Redução \\
\hline 2500 a $2999 \mathrm{~g}$ & $-0,6068$ & 0,3682 & $-7,4364$ & 0,063 & Redução \\
\hline 3000 a $3999 \mathrm{~g}$ & $-0,9126$ & 0,8328 & $-14,7758$ & 0,000 & Redução \\
\hline $4000 \mathrm{~g}$ e mais & $-0,5538$ & 0,3067 & $-1,6788$ & 0,097 & Redução \\
\hline
\end{tabular}

$\mathrm{r}=$ coeficiente de correlação de Pearson; $\mathrm{R}^{2}=$ coeficiente de regressão linear; $\beta$ = intercepto da reta $\mathrm{X}$ com o eixo $\mathrm{Y}$ da regressão linear; $\mathrm{p}$ valor = nível de significância da regressão linear. Fonte: Sistema de Informações sobre Mortalidade - SIM.

A mortalidade neonatal foi reduzida ao longo do período amostrado (2010-2019) em função da cor da pele/raça da mãe, com maior redução quando categorizado a cor/raça "não branca" da mãe $(\beta=-48,7818)$ em relação a cor/raça "branca" $(\beta$ = -20,6121) (Tabela 4). Em relação a idade da mãe, reduções na mortalidade neonatal foram observadas na faixa etária de 10 a 19 anos $(\beta=-22,0424)$ e 20 a 29 anos $(\beta=-26,8061)$. No entanto, tendência de aumento foi constatado para mães com idade entre 30 e 39 anos $(\beta=7,5333)$ e acima de 40 anos $(\beta=1,5818)$ (Tabela 4$)$. 
Tabela 4. Tendência temporal da mortalidade neonatal no Estado do Pará entre 2010 e 2019, em função da cor da pele/raça e idade da mãe.

\begin{tabular}{|c|c|c|c|c|c|}
\hline Cor da pele/raça & $\mathrm{r}$ & $\mathrm{R}^{2}$ & $\beta$ & p-valor & Tendência \\
\hline Branca & $-0,9337$ & 0,8717 & $-20,6121$ & $<0,001$ & Redução \\
\hline Não branca & $-0,9422$ & 0,8878 & $-48,7818$ & $<0,001$ & Redução \\
\hline Idade & $\mathrm{r}$ & $\mathrm{R}^{2}$ & $\beta$ & p-valor & Tendência \\
\hline 10 a 19 anos & $-0,9642$ & 0,9297 & $-22,0424$ & $<0,001$ & Redução \\
\hline 20 a 29 anos & $-0,8855$ & 0,7841 & $-26,8061$ & $<0,001$ & Redução \\
\hline 30 a 39 anos & 0,6194 & 0,3837 & 7,5333 & 0,056 & Aumento \\
\hline Acima de 40 anos & 0,5547 & 0,3077 & 1,5818 & 0,096 & Aumento \\
\hline
\end{tabular}

$r=$ coeficiente de correlação de Pearson; $R^{2}=$ coeficiente de regressão linear; $\beta$ = intercepto da reta $X$ com o eixo $Y$ da regressão linear; $p$ valor $=$ nível de significância da regressão linear. Fonte: Sistema de Informações sobre Mortalidade - SIM.

Ao analisar a duração da gestação em semanas (menos de 22, 28 a 31, 32 a 36, 37 a 41 e 42 semanas ou mais), o tipo de gravidez (única, dupla e tripla ou mais) e o tipo de parto (vaginal ou cesárea), é possível observar que houve uma redução na mortalidade neonatal em todas essas categorias supramencionadas. As maiores reduções foram obtidas para duração da gestação entre 37 e 41 semanas $(\beta=-32,2848)$, tipo de gravidez única $(\beta=-39,5879)$, e tipo de parto vaginal $(\beta=-39,5879)$. Destaca-se também as menores reduções na mortalidade neonatal no período estudado, as quais foram obtidas para a duração da gestação entre 28 e 31 semanas $(\beta=-0,9939)$, tipo de gravidez dupla $(\beta=--0,9636)$ e tipo de parto cesariana $(\beta=-2,4667)$. O único aumento obtido na mortalidade neonatal no Estado do Pará, entre 2010 e 2019, foi para a duração da gestação entre 22 e 37 semanas $(\beta=1,8000)$ (Tabela 5$)$.

Tabela 5. Tendência temporal da mortalidade neonatal no Estado do Pará entre 2010 e 2019, em função da duração da gestação, tipo de gravidez e tipo de parto.

\begin{tabular}{|c|c|c|c|c|c|}
\hline Duração da gestação & $\mathrm{r}$ & $\mathrm{R}^{2}$ & $\beta$ & $\mathrm{p}$-valor & Tendência \\
\hline Menos de 22 semanas & $-0,2513$ & 0,0632 & $-2,8606$ & 0,484 & Redução \\
\hline 22 a 37 semanas & 0,2802 & 0,0785 & 1,8000 & 0,433 & Aumento \\
\hline 28 a 31 semanas & $-0,1646$ & 0,0271 & $-0,9939$ & 0,650 & Redução \\
\hline 32 a 36 semanas & $-0,8097$ & 0,6556 & $-8,3394$ & 0,005 & Redução \\
\hline 37 a 41 semanas & $-0,7811$ & 0,6101 & $-32,2848$ & 0,008 & Redução \\
\hline 42 semanas e mais & $-0,6856$ & 0,4701 & $-3,5152$ & 0,029 & Redução \\
\hline Tipo de gravidez & $\mathrm{r}$ & $\mathrm{R}^{2}$ & $\beta$ & p-valor & Tendência \\
\hline Única & $-0,9053$ & 0,8197 & $-39,5879$ & $<0,001$ & Redução \\
\hline Dupla & $-0,4382$ & 0,1921 & $-0,9636$ & 0,205 & Redução \\
\hline Tripla ou mais & $-0,8530$ & 0,7276 & $-1,1879$ & 0,002 & Redução \\
\hline Tipo de parto & $\mathrm{r}$ & $\mathrm{R}^{2}$ & $\beta$ & p-valor & Tendência \\
\hline Vaginal & $-0,8623$ & 0,7435 & $-39,5879$ & 0,001 & Redução \\
\hline Cesárea & $-0,3573$ & 0,1276 & $-2,4667$ & 0,311 & Redução \\
\hline
\end{tabular}

$\mathrm{r}=$ coeficiente de correlação de Pearson; $\mathrm{R}^{2}=$ coeficiente de regressão linear; $\beta$ = intercepto da reta $\mathrm{X}$ com o eixo $\mathrm{Y}$ da regressão linear; $\mathrm{p}$ valor = nível de significância da regressão linear. Fonte: Sistema de Informações sobre Mortalidade - SIM. 


\section{Discussão}

A mortalidade infantil (MI) é classicamente considerada um dos melhores indicadores do nível de vida e bem-estar social de uma população (Araujo Filho et al., 2017). Este evento representa um episódio indesejável em saúde pública, pois são mortes precoces, e em sua maioria, evitáveis. Note que dentre as 13 regiões de saúde do Estado do Pará, caracterizadas no presente estudo, 10 regiões (76,92\%) obtiveram uma tendência temporal na mortalidade neonatal entre 2010 e 2019 , enquanto 3 regiões $(23,08 \%)$ apresentaram uma tendência de aumento ao longo deste período. Outros estudos também corroboram com está tendência de redução, ao longo dos anos, na mortalidade neonatal em diversas regiões do Brasil, a exemplo de estudos desenvolvidos na cidade de Recife, Pernambuco (Oliveira et al., 2016); em Salvador, Bahia (Gonçalves et al., 2015); no Distrito Federal (Monteiro \& Schmitz, 2007); e no Nordeste brasileiro (Santos et al., 2019).

Em um estudo realizado no Pará, no período de 1996 a 2017, foi constatado um declínio nos patamares de mortalidade neonatal precoce no estado, ficando inclusive com nível inferior ao recomendado nos Objetivos de Desenvolvimento Sustentável (ODS) 2030 do Brasil, que é de 12/1000 nascidos vivos (Pontes et al., 2020). Estes resultados indicam uma evolução positiva da saúde pública no estado do Pará, o que é consistente com o que foi relatado no presente capítulo e pode ser decorrente da implementação de políticas voltadas à gestante e ao neonatal, como a implementação da Rede Cegonha, associada à melhoria da qualidade de vida de grupos socioeconomicamente mais vulneráveis (Barros \& Albernaz, 2011; Malta et al., 2010).

Ao observar que a tendência da mortalidade neonatal, em função do sexo da criança, foi reduzida em sua totalidade, com maiores reduções em crianças do sexo masculino (Tabela 2), não é condizente com outros estudos, que relataram probabilidade de morte para o sexo masculino sendo relativamente maior do que para o sexo feminino, assim como a probabilidade de morte diminui significativamente com o aumento da idade (Ortiz \& Oushiro, 2008; Sousa et al., 2019). Estudos realizados anteriormente no estado do Pará, que avaliou a distribuição dos óbitos neonatais de 1996 a 2017 (Pontes et al., 2020) e a relação entre mortalidade infantil e doenças infecciosas e parasitárias (Sousa et al., 2019), foi relatado uma maior taxa de mortalidade neonatal para o gênero masculino (60\% dos óbitos) e um maior risco de óbito no período neonatal precoce (de 0 a 6 dias após o nascimento). No entanto, foi constatado no presente estudo que houve uma maior redução na mortalidade neonatal para o sexo masculino e para recém-nascidos com faixa etária menos tardia, evidenciando que estes fatores não se caracterizam como um risco para a mortalidade neonatal no Estado do Pará.

Em relação ao peso da criança ao nascer, os resultados expostos na Tabela 3 vão de encontro a outros relatos feitos na bibliografia, os quais constataram que quanto menor o peso da criança ao nascer, maior a possibilidade de evolução desfavorável devido a complicações pós-natais (Barbeiro et al., 2015; França et al., 2017). Pesquisas sugerem que o baixo peso ao nascer é um sinal de risco social e está relacionado as condições socioeconômicas instáveis e comportamento materno relacionado aos cuidados de saúde (Walther, 2005). Isso porque os recém-nascidos de baixo peso têm maior probabilidade de desenvolver pulmões imaturos e distúrbios metabólicos, levando ou agravando a patologia que os acomete, aumentando o risco de morbimortalidade (França \& Lansky, 2016). Deste modo, o risco relativo durante a primeira semana de vida chega a ser 165 vezes maior que o correspondente aos nascidos com peso adequado, relação que diminui para 132 vezes nas semanas seguintes (Ortiz \& Oushiro, 2008).

No que tange a questão racial da mãe, é interessante destacar que para esta variável foi considerado como "não branca" todas as outras categorias de classificação utilizado pelo IBGE, que são: preta, parda, amarela e indígena (Petruccelli \& Sabola, 2013). Deste modo, apesar de outros trabalhos relatarem maior número de óbitos neonatais em mães com cor/raça "não branca", principalmente na população indígena (Sousa et al., 2019), o que fica evidente no presente estudo é que as condições de vida relacionada a economia, cultura, condições sociais e étnicas, têm refletido positivamente para reduzir as mortalidades neonatais no Estado do Pará, miscigenando a população e reduzindo as "diferenças" sociais. 
Ainda sobre as variáveis relacionadas à mãe, o resultado obtido para a categoria idade materna acima de 30 anos (Tabela 4), demonstra ser um fator de risco para desfechos negativos da gestação, principalmente mães com idade acima de 40 anos. A idade materna é considerada uma variável importante na determinação da mortalidade neonatal, pois os extremos de idade para concepção apresentam maiores chances de complicações e consequente risco de morte (Lima et al., 2012). Alguns estudos destacam a idade materna inferior a 20 anos ou superior a 35 anos, como sendo uma das principais causas da mortalidade neonatal (Almeida et al., 2002; Haiddar et al., 2001). No entanto, no presente estudo a idade inferior a 20 anos, não caracterizou um fator de risco para a mortalidade neonatal, ocorrendo até uma redução nas taxas de mortalidades em mães de 10 a 29 anos.

Houve uma tendência de aumento na mortalidade neonatal no Estado do Pará, entre 2010 e 2019, para a categoria da duração gestacional entre 22 e 37 semanas (Tabela 5). Em estudos de sobrevivência infantil, a duração da gestação é considerada um indicador da viabilidade do recém-nascido (Ferrari et al., 2006; Kilsztajn et al., 2003). Assim, a duração da gestação representa o componente mais importante do desenvolvimento intrauterino, identificando a prematuridade (gestações com duração inferior a 37 semanas) como um dos principais riscos de mortalidade neonatal (Ortiz \& Oushiro, 2008).

Estudo realizado no Estado do Pará, o qual analisou a mortalidade neonatal no Estado Paraense no período compreendido entre 1996 e 2017, os autores declaram que o óbito neonatal precoce no estado do Pará ainda é considerado elevado, embora já existam políticas púbicas de atenção à saúde materno-infantil (Pontes et al., 2020). No entanto, os dados do presente estudo revelam que a tendência temporal da mortalidade neonatal em um período compreendido de 10 anos (2010 a 2019), houve uma maior redução de óbitos neonatais do que casos de aumento neste período estudado.

No geral, as variáveis relacionadas à gravidez (duração da gestação, tipo de gravidez e tipo de parto) não caracterizam um fator substancial de risco para a mortalidade neonatal no Estado do Pará, com exceção da duração da gestação entre 22 e 37 semanas, que apresentou aumento na mortalidade neonatal. Ademais, outras variáveis que podem ser destacadas e estão relacionadas diretamente com o risco de mortalidade neonatal no Estado do Pará, são: peso ao nascer até 500 g e idade da mãe acima de 30 anos.

\section{Considerações Finais}

Neste estudo foi encontrado significativas reduções da mortalidade neonatal no Estado do Pará, no período amostrado entre 2010 e 2019. Porém, apesar do decréscimo em todo o estado, é possível concluir que as regiões de saúde do Araguaia, Baixo Amazonas e Marajó II, necessitam de uma atenção especial por parte das autoridades governamentais, visto que estas apresentaram uma tendência de aumento na mortalidade neonatal.

Os riscos de morte mais elevados de neonatais no Estado do Pará, correspondem à fatores do peso ao nascer de até 500 g, faixa etária da mãe acima de 30 anos e duração da gestação entre 22 e 37 semanas. O presente estudo aponta para a necessidade de ampliar e intensificar a implementação de políticas e ações de saúde direcionadas para atenção pré-natal.

Como viés do estudo, foi encontrado muitos dados ignorados no Sistema de Informação. No entanto, as informações encontradas podem subsidiar a formulação de estratégias, com foco nas regiões de saúde e nos principais fatores de risco para a mortalidade neonatal em todo o Estado Paraense.

\section{Referências}

Almeida, M. F., Novaes, H. M. D., Alencar, G. P., \& Rodrigues, L. C (2002). Mortalidade neonatal no município de São Paulo: influências do peso ao nascer e de fatores sócio-demográficos e assistências. Revista Brasileira de Epidemiologia, 5 (1), 93-106.

Araujo Filho, A. C. A., Sales, I. M. M., Araújo, A. K. L., Almeida, P. D., \& Rocha, S. S (2017). Aspectos epidemiológicos da mortalidade neonatal em capital do nordeste do Brasil. Revista Cuidarte, 8 (3), 1767-1776. 
Barbeiro, F. M. S., Fonseca, S. C., Tauffer, M. G., Ferreira, M. S. S., Silva, F. P., Ventura, P. M., \& Quadros, J. I (2015). Óbitos fetais no Brasil: revisão sistemática. Revista de Saúde Pública, 49 (22), 1-15.

Barros, A. J. D., \& Albernaz, E (2011). Óbitos evitáveis até 48 meses de idade entre as crianças da coorte de nascimentos de Pelotas de 2004 . Revista de Saúde Pública, 45, 334-342.

Brasil. (2011). Portaria N $N^{o}$ 1.459, de 24 de junho de 2011. Sistema Único de Saúde - SUS - a Rede Cegonha. Brasília: Ministério da Saúde. <http://bvsms.saude.gov.br/bvs/saudelegis/gm/2011/prt1459_24_06_2011.html>.

Brasil. (2002). Rede Interagencial de Informações para a Saúde - Ripsa. Indicadores Básicos para a Saúde no Brasil: conceitos e aplicações. Organização PanAmericana de Saúde.

Ferrari, L. S. L., Brito, A. S. J., Carvalho, A. B. R., \& Gonzáles, M. R. C. (2006). Mortalidade neonatal no Município de Londrina, Paraná, Brasil, nos anos 1994, 1999 e 2002. Cadernos de Saúde Pública, 22 (5), 1063-1071.

França, E. B., Lansky, S., Rego, M. A. S., Malta, D. C., França, J. S., Teixeira, R., Porto, D., Almeida, M. F., Souza, M. F. M., Szwarcwald, C. L., Mooney, M., Naghavi, M., \& Vasconcelos, A. M. N (2017). Principais causas da mortalidade na infância no Brasil, em 1990 e 2015: estimativas do estudo de Carga Global de Doença. Revista Brasileira de Epidemiologia, 20 (1), 46-60.

França, E \& Lansky, S (2016). Mortalidade infantil neonatal no Brasil: situação, tendências e perspectivas. <http://www.abep.org.br/publicacoes/index.php/anais/article/view/1763>.

Gaiva, M. A. M., Fujimori, E., \& Sato, A. P. S (2015). Mortalidade neonatal: análise das causas evitáveis. Revista Enfermagem UERJ, 23 (2), $247-253$.

Gonçalves, A. C., Costa, M. C. N., Barreto, F. R., Paim, J. S., Nascimento, E. M. R., Paixão, E. S., \& Mota, E. L. A (2015). Tendência da mortalidade neonatal na cidade de Salvador (Bahia-Brasil), 1996-2012. Revista Brasileira de Saúde Materno Infantil, 15 (3), $337-347$.

Haiddar, F. H., Oliveira, U. F., \& Nascimento, L. F. C (2001). Escolaridade materna: correlação com os indicadores obstétricos. Cadernos de Saúde Pública, 17 (4), 1025-1029.

IBGE. (2021). Instituto Brasileiro de Geografia e Estatística. Taxa de mortalidade infantil. <https://cidades.ibge.gov.br/brasil/pa/pesquisa/39/ 30279?localidade1=13\&tipo=rankin>.

Kilsztajn, S., Rossbach, A., Carmo, M. S. N., \& Sugahara, G. T. L. (2003). Assistência pré-natal, baixo peso e prematuridade no Estado de São Paulo, 2000. Revista de Saúde Pública, 37 (3), 303-310.

Kropiwiec, M. V., Franco, S. C., \& Amaral, A. R (2017). Fatores associados à mortalidade infantil em município com índice de desenvolvimento humano elevado. Revista Paulista de Pediatria, 35 (4), 391-398.

Lima, E. F. A., Sousa, A. I., Griep, R. H., \& Primo, C. C. (2012). Fatores de risco para mortalidade neonatal no município de Serra, Espírito Santo. Revista Brasileira de Enfermagem, 65 (4), 578-585.

Malta, D. C., Duarte, E. C., Escalante, J. J. C., Almeida, M. F., Sardinha, L. M. V., Macário, E. M., Monteiro, R. A., \& Morais Neto, O. L (2010). Mortes evitáveis em menores de um ano, Brasil, 1997 a 2006: contribuições para a avaliação de desempenho do Sistema Único de Saúde. Cadernos de Saúde Pública, $26(3), 481-491$.

Migoto, M. T., Oliveira, R. P., Silva, A. M. R., \& Freire, M. H. S. (2018). Mortalidade neonatal precoce e fatores de risco: estudo caso-controle no Paraná. Revista Brasileira de Enfermagem, 7 (15). 2675-2683.

Monteiro, R. A., \& Schmitz, B. A. S. (2007). Infant mortality in the Federal District, Brazil: time trend and socioeconomic inequalities. Cadernos de Saúde Pública, 23 (4), 767-774.

Oliveira, C. M., Bonfim, C. V., Guimarães, M. J. B., Frias, P. G., \& Medeiros, Z. M. (2016). Mortalidade infantil: tendência temporal e contribuição da vigilância do óbito. Acta Paulista de Enfermagem, 29 (3), 282-290.

Ortiz, L. P., \& Oushiro, D. A. (2008). Perfil da mortalidade neonatal no Estado de São Paulo. São Paulo em Perspectiva, 22 (1), $19-29$.

Paim, J., Travassos, C., Almeida, C., Bahia, L., \& Macinko, J. (2011). O sistema de saúde brasileiro: história, avanços e desafios. Lancet, 11, 60054-60058.

Petruccelli, J. L. \& Sabola, A. L. (2013). Características étnico-raciais da população: classificações e identidades. Rio de Janeiro: Instituto Brasileiro de Geografia e Estatística - IBGE, 208p.

Pontes, M. A. G., Nascimento, F. A. S., Silva, D. F. A., Silva, M. L., Cunha, K. C., \& Chermont, A. G. (2020). Mortalidade neonatal precoce no estado do Pará, Região Amazônica do Brasil. Revista CPAQV, 12 (2), 1-16.

Santos, A. D., Santos, M. B., Lima, S. V. M. A., \& Santos, R. J. (2019). Temporal trends of mortality in the city of Lagarto, Northeast of Brazil. Revista de Pesquisa Cuidado é Fundamental, 11 (5), 1155-1160.

Silveira, M. F., Barros, A. J. D., Santos, I. S., Matijasevich, A., \& Victoria, C. G. (2008). Diferenciais socioeconômicos na realização de exame de urina no pré-natal. Revista de Saúde Pública, 42 (3), 389-395.

Soares, E. S., \& Menezes, G. M. S. (2010). Fatores associados à mortalidade neonatal precoce: análise de situação no nível local. Epidemiologia e Serviços de Saúde, 19 (1), 51-60.

Sousa, J. F., Santos, K. F., Santos, D. R., Silva, A. V. C., Pereira, I. S., \& Silva, R. C (2019). Mortalidade infantil por doenças infecciosas e parasitárias no estado do Pará: vigilância de óbitos entre 2008 a 2017. Pará Research Medical Journal, 3 (3-4), 1-8. 
Research, Society and Development, v. 10, n. 13, e595101321613, 2021

(CC BY 4.0) | ISSN 2525-3409 | DOI: http://dx.doi.org/10.33448/rsd-v10i13.21613

Tomio, G. O., \& Magajewski, F. (2019). Tendência temporal da mortalidade neonatal e sua relação com as variáveis associadas à desigualdade social em Santa Catarina no período de 2008 a 2016. Universidade do Sul de Santa Catarina. Disponível em: <https://www.riuni.unisul.br/handle/12345/7380>.

Veloso, F. C. S., Kasar, L. M. L., Oliveira, M. J. C., Lima, T. H. B., Bueno, N. B., Gurgel, R. Q., \& Kassar, S. B. Análise dos fatores de risco na mortalidade neonatal no Brasil: uma revisão sistemática e metanálise de estudos observacionais. Jornal de Pediatria, 95 (5), 519-530.

Viellas, E. F., Domingues, R. M. S. M., Dias, M. A. B., Gama, S. G. N., Theme Filha, M. M., Costa, J. V., Bastos, M. H., \& Leal, M. C. (2014). Assistência pré-natal no Brasil. Cadernos de Saúde Pública, 30, 85-100.

Walker, N., Yenokyan, G., Friberg, I. K., \& Bryce, J (2013). Patterns in coverage of maternal, newborn, and child health interventions: Projections of neonatal and under-5 mortality to 2035. The Lancet, 382 (9897), 1029-1038.

Walther, F. J (2005). Withholding treatment, withdrawing treatment, and palliative care in the neonatal intensive care unit. Early Human Development, 81 (12), 965-972. 